\title{
Hepatitis B Virus Core Promoter Mutations in Patients With Chronic Hepatitis B and Hepatocellular Carcinoma in Bucharest, Romania
}

\author{
Ileana Constantinescu ${ }^{1,2,} ;$ Andrei-Antoniu Dinu ${ }^{2}$; Voicu Boscaiu ${ }^{3}$; Marius Niculescu ${ }^{4}$ \\ ${ }_{1}^{1}$ Immunology of Transplantation Discipline, Faculty of Medicine, Carol Davila University of Medicine and Pharmacy, Bucharest, Romania \\ ${ }^{2}$ Center for Immunogenetics and Virology, Fundeni Clinical Institute, Bucharest, Romania \\ 3 “Gheorghe Mihoc-Caius Iacob” Institute of Statistics and Applied Mathematics, Bucharest, Romania \\ ${ }^{4}$ Colentina Clinical Hospital, Bucharest, Romania \\ *Corresponding Author: Ileana Constantinescu, Centre for Immunogenetics and Virology, Fundeni Clinical Institute, Bucharest, Romania. Tel: +40-744341984, Fax: +40-213180448, \\ E-mail: ileana.constantinescu@imunogenetica.ro
}

Received: July 25, 2014; Revised: September 10, 2014; Accepted: September 26, 2014

\begin{abstract}
Background: Accurate and personalized molecular virological diagnosis of hepatitis B virus (HBV) infection is crucial for individualized selection of patients for antiviral therapy in Romania.

Objectives: We aimed to investigate HBV mutations in Romanian patients with chronic HBV infection, also to match HBV genotypes with HBV mutations identified and clinical outcomes.

Patients and Methods: This was a cross-sectional study. A total of 484 Romanian patients with chronic HBV infection and hepatocellular carcinoma (HCC) were investigated. This was performed in Fundeni Clinical Institute, Bucharest, Romania during January 2005 to August 2010. HBsAg positive patients with chronic HBV infection admitted to Fundeni Clinical Institute were randomly enrolled in the study. Analysis was performed in the Centre for Immunogenetics and Virology, Fundeni Clinical Institute, Bucharest, Romania. Indirect diagnosis was performed with enhanced chemiluminescence method using Architect i2000SR and HBV-DNA was quantified with COBAS TaqMan HBV PCR. Direct sequencing of the PCR-products was performed with the PCR-product sequencing kit. HBV genotyping was performed with INNO-LiPA DR Amplification and INNO-LiPA HBV precore-core.

Results: We detected two HBV genotypes; $\mathrm{A}(8.1 \%)$ and $\mathrm{D}(60.5 \%)$, and a mixture of genotypes Aand $\mathrm{D}(31.4 \%)(\mathrm{P}<0.001)$. Basal core promoter (BCP) A1762T/G1764A and precore (PC) G1896A mutations were detected in these Romanian patients with chronic HBV infection. HBV chronic carriers had mainly genotype D (54.4\%) and HBVWT (64.0\%). BCP A1762T, G1764A and PC G1896A were significantly associated with HCC-tissue HBV sequencing (75.3\%) (P<0.001). PC G1896A alone was detected in HCC-serum HBV sequencing group (66.7\%).

Conclusions: Genotype D was the main genotype detected in Romanian patients with chronic HBV infection. Genotype D presented both $\mathrm{BCP}$ and PC mutations more frequently.
\end{abstract}

Keywords: Hepatitis B Virus; Hepatocellular carcinoma; Genotype; Mutations

\section{Background}

Worldwide, more than 500 million patients with chronic hepatitis B virus infection are at risk of developing liver cirrhosis or hepatocellular carcinoma (HCC) (1). This is a serious health problem. Romania is one of the SouthEastern European countries with a high prevalence of chronic hepatitis B virus (HBV) infection as 5.59\% in 2010, and a larger number of HBsAg positive people (2). Development of chronic HBV infection is dependent on the age of $\mathrm{HBV}$ exposure. It is estimated that $15-40 \%$ of chronic hepatitis B patients would progress to cirrhosis and HCC (2). The reasons why some patients with chronic HBV infection progress to HCC are under debates. Host factors such as immune response to $\mathrm{HBV}$, genetic predisposition to HCC development, high HBV replication rates, as well as certain mutations within the HBV genome have been related to HCC development. A dual mutation in the basal core promoter $(\mathrm{BCP})$ region of the HBV genome involving an A-T substitution at nucleotide 1762 and a G-A substitution at nucleotide 1764 has been associated with HCC development. Moreover, a mutation in the precore (PC) region of the HBV genome involving a G-A substitution at nucleotide 1896 has been described in HBeAgnegative patients. However, the role of these mutations in HCC pathogenesis is not well established (3). The PC region mutations prevent the production of hepatitis $B$ e antigen (HBeAg) by introducing a premature stop codon into the open reading frame of the precore region. Another common mutation is a double mutation in $\mathrm{BCP}$ region (A1762T/G1764A), which aborts the transcription of precore mRNA, but does not seriously affect the viral pregenome RNA, resulting in a decrease of HBeAg expression up to $70 \%$ and enhanced viral genome replication. Although the frequencies of precore and BCP mutations vary between HBV genotypes, they are associated with fulminant hepatitis $B$ and hepatocellular carcinoma, in quite a few cases (4). The level of circulating

Copyright (C) 2014, Kowsar.; Published by Kowsar. This is an open-access article distributed under the terms of the Creative Commons Attribution-NonCommercial 4.0 International License (http://creativecommons.org/licenses/by-nc/4.0/) which permits copy and redistribute the material just in noncommercial usages, provided the original work is properly cited. 
viremia is a risk factor for development of cirrhosis and HCC, and is used to diagnose hepatitis B infection and define response to antiviral therapy. Since chronic hepatitis, cirrhosis and HCC have been found in patients with low serum HBV DNA-levels, the current cutoff value of $20.000 \mathrm{IU} / \mathrm{mL}$ is still debatable. Further understanding of virological characteristics in patients with low HBVDNA levels would be helpful for clinical decisions in management of patients with low circulating viremia. Many studies have demonstrated that HBV genotype, precore and $\mathrm{BCP}$ mutations have a substantial impact on progression of chronic hepatitis B toward HCC (4). Currently, ten genotypes of HBV (A-J) have been identified, based upon a divergence of $8 \%$ or more across the entire nucleotide sequence $(4,5)$. Sequence differences between HBV genotypes can lead to structural differences at the level of pregenome and can lead to important differences at the translational level when specific and common mutations $\operatorname{occur}(6,7)$.

\section{Objectives}

It is of great interest to study Romanian HBV variants, to investigate the precore-core promoter mutations to have a personalized viral diagnosis, for a better response to therapy and clinical outcome. In addition, to sequence HBV genome of nonresponders Romanian patients to antiviral therapy to see if there is a link between HBV mutations and lack of both, biochemical and virological responses in these patients. Another aspect of interest is HCC onset within chronic hepatitis B long carrier status. No research has been performed in our country on chronic HBV-HCC Romanian patients. Therefore, it would be important to sequence HBV-DNA from HBV chronic carriers, HBV chronic carriers coinfected with HDV, HCC patients' tissue and serum samples to investigate mutations in the core promoter region of the HBV genome.

\section{Patients and Methods}

\subsection{Selection of Patients}

This cross-sectional investigation was performed in Fundeni Clinical Institute, Bucharest, Romania during January 2005 and August 2010. HBsAg positive patients with chronic HBV infection admitted to Fundeni Clinical Institute were randomly enrolled in the study. We assessed 484 positive patients. Randomly, we obtained the following patient diagnosis status: $375 \mathrm{HBV}$ chronic carriers, 94 HCC and 15 HBV chronic carriers coinfected with HDV. All HBV chronic carriers and all HBV chronic carriers coinfected with HDV were detected by serum analysis. Nonetheless, regarding HCC patients, the first 73 were described by tissue HBV sequencing and the last 21 by serum analysis. Diagnosis of HCC was confirmed by biopsy of the mass lesion in the liver, and by typical computed tomography or magnetic resonance imaging findings for HCC. Informed consent was obtained from all patients. Patient privacy was respected over the testing and analyzing data. The Ethics Committee of the Fundeni Clinical Institute, Bucharest, Romania approved the study (2010.08.30). All data remained confidential.

\subsection{Laboratory Tests}

\subsubsection{Serological Markers}

For indirect diagnosis, sera obtained for all patients were tested for HBsAg and HbeAg and anti-HBs, anti-HBe and total anti-HBc antibodies using automated Architect i2000SR (Abbott) by chemiluminescence. For anti-HDV antibodies, we used a competitive ELISA method (Medical Biological Service, Italy).

\subsubsection{HBV DNA Quantification}

HBV-DNA was quantified by a real-time polymerase chain reaction assay in the linear range from $10^{2}$ to $10^{11} \mathrm{cop}$ ies/mL using COBAS TaqMan HBV test (Roche).

\subsubsection{HBV Sequencing}

Detection of BCP mutations, PC mutations and HBV genotypes was performed using Revers Hybridization Line Probe Assays-INNO-LiPA HBV DR V2 and INNO-LiPA HBV precore-core (Innogenetics, Ghent. Belgium). For all patients, we performed HBV sequencing: for 73 HCC patients tissue HBV sequencing, 21 HCC patients serum HBVsequencing and for chronic patients serum sequencing. In chronic patients, viral DNA was obtained from sera. For 73 HBV associated HCC patients, tumor tissue samples were obtained and analyzed.

\subsection{Statistical Analysis Tools}

Pearson $\chi 2$ test was used to verify the independence of two discrete variables in contingency tables. To compare the distribution of serum DNA HBV for considered factors (diagnosis, HBV genotype and mutation) we used a univariate General Linear Model and One-way ANOVA test with Tamhane test. In testing and graph representation, we used natural logarithm of serum DNA-HBV instead of genuine variable.

\section{Results}

We detected two genotypes of $\mathrm{A}$ and $\mathrm{D}$ in our study population, as follows: A (i.e. patient has only genotype A), D (i.e. patient has only genotype D), A+D (i.e. patient has both genotypes $A$ and $D$, higher concentration of genotype $\mathrm{D}$ ). The decreasing order of HBV genotypes prevalence was $\mathrm{D}, \mathrm{A}+\mathrm{D}, \mathrm{A}$. In our sample, the prevalence rates were $60.5 \%, 31.4 \%$ and $8.1 \%$ for $\mathrm{D}, \mathrm{A}+\mathrm{D}$ and $\mathrm{A}$, respectively. The differences were significant $\left(\mathrm{P}<0.001\right.$ for $\chi^{2}$ Pearson Test; see last column of Table 1).

HBV genotype and diagnosis were dependent variables 
( $\mathrm{P}<0.001$ for Pearson $\chi^{2}$ test). The minimum value of the prevalence of genotype D reached $54.4 \%$ for chronic carriers, which was significantly smaller than other diagnoses $\left(P<0.050\right.$ for $\chi^{2}$ Pearson Test). Conversely, for chronic carriers the genotype combination $\mathrm{A}+\mathrm{D}$ had the highest prevalence (37.6\%, compared to about $10 \%$, for other diagnoses). While, the prevalence of genotype A was not significantly dependent on diagnosis (Table 1). In 152 patients we detected both genotypes A and D, with a higher prevalence in chronic carriers (141 patients, 37.6\%), followed by HCC-tissue sample HBV sequencing group (7 patients, 9.6\%), HCC-serum HBV sequencing group (3 patients, $14.6 \%$ ) and chronic carriers with HDV coinfection (one patient, 6.7\%). HBV genotype and diagnosis were dependent ( $P<0.001$ for Pearson $\chi^{2}$ test). Definitely, distribution of HBV genotype for chronic carriers was significantly different from the distribution of HCC-tissue HBV sequencing $\left(\mathrm{P}<0.001\right.$ for Pearson $\chi^{2}$ test $)$ and chronic carriers + $\operatorname{HDV}\left(\mathrm{P}=0.012\right.$ for Pearson $\chi^{2}$ test $)$. To be more specific, the prevalence of $\mathrm{A}+\mathrm{D}$ was $37.6 \%$ for chronic carriers, but significantly smaller for HCC-tissue HBV sequencing (9.6\%) and chronic carriers + HDV (6.7\%). Conversely, D prevalence was higher for HCC-tissue HBV sequencing (82.2\%) and chronic carriers + HDV (93.3\%), compared to chronic carriers (54.4\%) (see Table 1 ).

We next analyzed the presence of BCP and PC mutations (see last column of Table 1). BCP mutations were reported for $34.9 \%(12.8 \%+22.1 \%)$ of patients and PC mutations for $36.4 \%(14.3 \%+22.1 \%)$. BCP and PC mutations were depen-
$\operatorname{dent}\left(\mathrm{P}<0.001\right.$ for $\chi^{2}$ Pearson Test of independence). Definitely, patients having both or non-BCP and PC mutations were $22.1 \%$ and $50.8 \%$, respectively (higher than expected based on the hypothesis of independence). Distribution of BCP and PC mutations were significantly linked to the viral diagnosis, excepting HCC-serum HBV sequencing and chronic carriers + HDV, which seem to have the same distribution of mutations (To be more specific, see Table 1). 1) WT was mainly associated with chronic carriers ( $64 \%$ of patients had WT); 2) BCP A1762T, G1764A and PC G1896A were significantly associated with HCC-tissue HBV sequencing (75.3\%); 3) PC G1896A was mainly associated with HCC-serum HBV sequencing and chronic carriers + HDV (66.7\% and respectively $73.3 \%$ of patients had PC G1896A). We had 39 patients with genotype A, 293 patients with genotype D and 152 with genotypes A+D. Patients with genotype A had mainly WT sequence (21 patients, $53.8 \%$ ). BCP mutation was detected in 12 patients (30.8\%), both BCP and PC mutation in six patients (15.4\%) and no PC mutation alone (See Table 2).

Distribution of BCP and PC mutations were significantly linked to genotypes $\left(\mathrm{P}<0.001\right.$ for $\chi^{2}$ Pearson Test of independence). Indeed, see Table 2.1) A+D genotypes combination was mainly associated with WT $(80.9 \%$ of patients with A+D had WT); 2) D genotype was mainly associated with presence of PC G1896A (50.1\% from D-patients present PC G1896A or BCP A1762T, G1764A-PC G1896);3) A genotype was mainly associated with presence of WT and BCP (the occurrence percentages were $53.8 \%$ and $30.8 \%$, respectively).

Table 1. Virologic Characteristics of Hepatitis B Virus Chronic Carriers and Patients With Hepatocellular Carcinoma ${ }^{\text {a,b }}$

\begin{tabular}{|c|c|c|c|c|c|}
\hline & $\begin{array}{l}\text { Chronic carriers } \\
\qquad \mathbf{n}=375\end{array}$ & $\begin{array}{c}\text { HCC-tissue } \mathrm{HBV} \\
\text { sequencing } n=73\end{array}$ & $\begin{array}{c}\text { HCC-serum HBV } \\
\text { sequencing } n=21\end{array}$ & $\begin{array}{c}\text { Chronic carriers } \\
+ \text { HDVn }=15\end{array}$ & Total $n=484$ \\
\hline Male & $196(52.27)$ & $38(52.05)$ & $12(57.14)$ & $9(60)$ & \\
\hline Age & $40.7 \pm 15.4$ & $51.4 \pm 17.2$ & $52.3 \pm 18.6$ & $46.1 \pm 13.9$ & \\
\hline \multicolumn{6}{|c|}{ HBV genotype Frequency } \\
\hline A & $30(8.0)$ & $6(8.2)$ & $3(14.3)$ & $0(0.0)$ & $39(8.1)$ \\
\hline $\mathrm{D}$ & $204(54.4)$ & $60(82.2)$ & $15(71.4)$ & $14(93.3)$ & $293(60.5)$ \\
\hline $\mathrm{A}+\mathrm{D}$ & $141(37.6)$ & $7(9.6)$ & $3(14.3)$ & $1(6.7)$ & $152(31.4)$ \\
\hline
\end{tabular}

Mutation Core/Precore

Frequency

\begin{tabular}{|c|c|c|c|c|c|}
\hline WT & $240(64.0)$ & $4(5.5)$ & $2(9.5)$ & $0(0.0)$ & $246(50.8)$ \\
\hline BCP A1762T,G1764A & $45(12.0)$ & $14(19.2)$ & $2(9.5)$ & $1(6.7)$ & $62(12.8)$ \\
\hline PC G1896A & 44 (11.7) & $0(0.0)$ & $14(66.7)$ & $11(73.3)$ & $69(14.3)$ \\
\hline $\begin{array}{l}\text { BCP A1762T,G1764A PC } \\
\text { G1896A }\end{array}$ & $46(12.3)$ & $55(75.3)$ & $3(14.3)$ & $3(20.0)$ & $107(22.1)$ \\
\hline
\end{tabular}

\section{DNA HBV}

\begin{tabular}{|c|c|c|c|c|}
\hline $\mathrm{Ln}, \mathrm{IU} / \mathrm{mL}$ & $10.49 \pm 3.45$ & $16.15 \pm 1.69$ & $13.47 \pm 1.22$ & $14.70 \pm 2.05$ \\
\hline
\end{tabular}

\footnotetext{
a Abbreviations: HCC, hepatocellular carcinoma; HBV, Hepatitis B Virus; HDV, Hepatitis D Virus; WT, Wild Type; BCP, Basal core promoter
}

${ }^{\mathrm{b}}$ Data are presented as Mean \pm SD or No. (\%). 
Constantinescu I et al.

\begin{tabular}{lccccc}
\hline Table 2. Mutation Characteristics of Hepatitis B Virus $\mathrm{a}, \mathrm{b}$ & & & \\
\hline Mutation & WT $\mathbf{n}=\mathbf{2 4 6}$ & $\begin{array}{c}\text { BCP A1762T,G1764A } \\
\mathbf{n}=\mathbf{6 2}\end{array}$ & PC G1896A $\mathbf{n}=\mathbf{6 9}$ & $\begin{array}{c}\text { BCP A1762T,G1764A } \\
\text { PC G1896A } \mathbf{n}=\mathbf{1 0 7}\end{array}$ & Total n= 484 \\
\hline HBV genotype Frequency & & & & & \\
\hline A & $21(53.8)^{\mathrm{C}}$ & $12(30.8)$ & $0(0.0)$ & $6(15.4)$ & $39(100)$ \\
D & $102(34.8)$ & $44(15.0)$ & $64(21.8)$ & $83(28.3)$ & $293(100)$ \\
A+D & $123(80.9)$ & $6(3.9)$ & $5(3.3)$ & $18(11.8)$ & $152(100)$
\end{tabular}

DNA HBV
\begin{tabular}{llllll} 
Ln, IU $/ \mathrm{mL}$ & $8.99 \pm 3.06$ & $14.44 \pm 1.99$ & $12.75 \pm 1.83$ & $15.23 \pm 2.16$ & $11.61 \pm 3.79$ \\
\hline
\end{tabular}

${ }^{\mathrm{a}}$ Abbreviations: WT, Wild Type; BCP, Basal core promoter; HBV, hepatitis B virus

$\mathrm{b}$ Data are presented as mean \pm SD or No. (\%).

${ }^{\mathrm{C}}$ Cells are frequencies. From a total of 39 patients with genotype A, 21 (namely 53.8\%) had WT sequence.

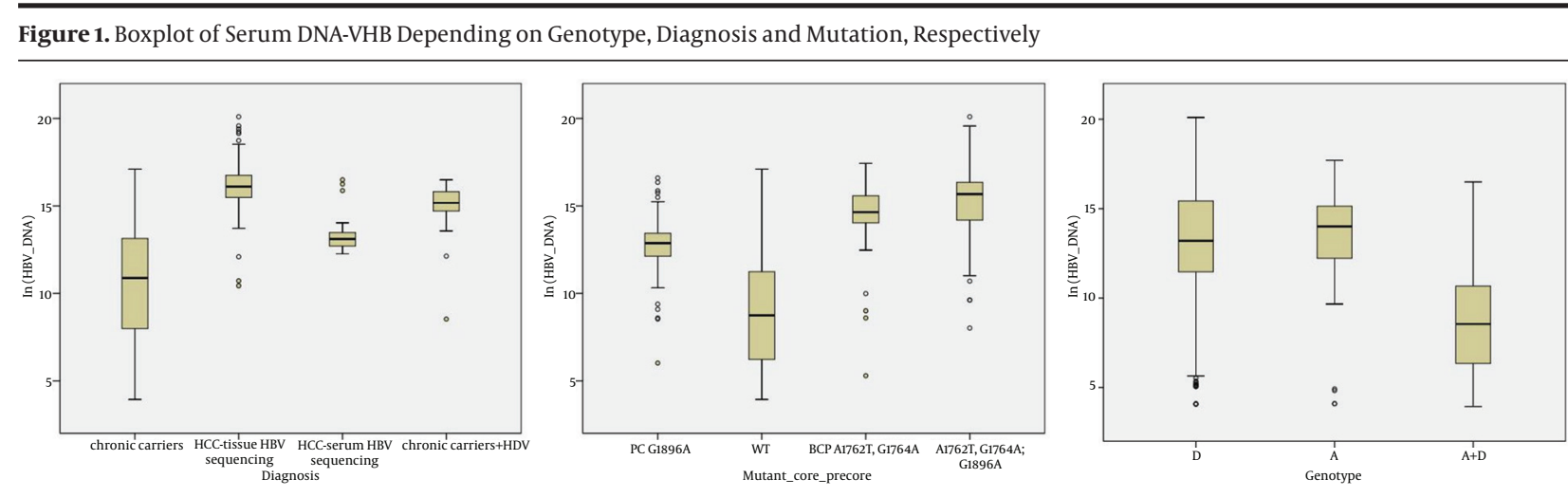

The isolated dots represent atypical values. Comment: boxplot for mutation reveals that, in spite of central tendency, the values of serum HBV DNA for mutations could be similar to WT.

One hundred and two patients (34.8\%) with genotype D had WT sequence, 44 (15.0\%) had BCP mutation only, $64(21.8 \%)$ had PC mutation only and $83(28.3 \%)$ had both BCP and PC mutations. WT sequence was mainly associated with genotype $\mathrm{A}+\mathrm{D}$ (123 patients, $80.9 \%)$. Analysis of variance was performed to study the dependence of the level of HBV DNA by factors genotype, diagnosis and mutation. We used the GLM Univariate procedure UNIANOVA of SPSS, which is able to consider both balanced and unbalanced models (our model was unbalanced and we lost test power). Using this GLM procedure, interactions between our factors were not significant but the effects of each individual factor were significant (for each factor F test had $\mathrm{P}<0.001$ ). Hence, we authorized to use post hoc One-way ANOVA procedure for each factor (we would apply Tamhane test). The mean serum HBV DNA values for the HCC-tissue HBV sequencing patients were significantly higher compared to chronic carriers (16.14 vs. 10.49; P $<0.001$ for Tamhane test; see (Table 1). Furthermore, HBV DNA values for the HCC-serum HBV sequencing group were lower than the HCC-tissue sequencing group (16.14 vs. $13.47, \mathrm{P}=0.004$; see Table 1 ). Genotypes $\mathrm{A}+\mathrm{D}$ was associated with lower mean of serum HBV DNA compared to genotypes D and A (8.89 vs. 12.82 and 13.01; Figure 1). The mean and median of serum HBV DNA were practically equal for Genotypes D and A, but significantly higher compared to $A+D(P<0.001)$. We detected significantly higher level of HBV DNA in serum of patients with both BCP A1762T/G1764A , PC G1896A mutation (15.23) and BCP A1762T/G1764A mutations (14.44) compared to patients with PC G1896A mutation alone (12.75) and patients with WT sequence (8.99); $\mathrm{P}<0.001$. Characteristics of chronic carriers were smallest DNA-HBV, predominance of WT and important percent of D presence, but also an important percent of $\mathrm{A}+\mathrm{D}$ presence. Characteristics of chronic carriers + VHD were high level of DNA-HBV, predominance of PC G1896A mutation and highest percent of genotype D presence. HCC characteristics were high level of DNA$\mathrm{HBV}$ and high percent of D presence. As it concerns mutations, there was an important difference between serum and tissue sequencing. Indeed, for serum sequencing PC G1896A was predominant, while for tissue sequencing BCP A1762T/G1764A and PC G1896A were predominant.

\section{Discussion}

We aimed to investigate genotype and mutations in 
Constantinescu I et al.

core promoter region of HBV genome in Romanian HBV clinical infected patients with chronic HBV hepatitis and HCC patients, for a better understanding of the presence of HBV genotypes and $\mathrm{BCP} / \mathrm{PC}$ mutations in this population. This paper was important because such a study has not been conducted in Romania. HBV genotyping was not performed in Romania in a group of patients of this size. This study helps to understand the evolution of HBV in patients with hepatitis $B$ and provides information about HBV genotypes and mutations. Previous studies have shown that patients infected with HBV genotype A had a higher sustained response to interferon therapy compared to HBV genotype D. Genotype D was predominant in Romania and previous studies have shown that patients with hepatitis $\mathrm{B}$ genotype $\mathrm{D}$ respond well to treatment with nucleoside analogue reverse transcriptase inhibitor $(8,9)$. Our study showed that knowing the genotype could be useful to predict the outcome of antiviral therapy in patients with chronic hepatitis B, but further studies are required to include a more rigorous selection of patients. Recent studies have shown a link between the presence of $\mathrm{BCP} / \mathrm{PC}$ mutations and hepatitis B-related acute-on-chronic liver failure. Patients infected with genotype $\mathrm{B}$ and both mutations $\mathrm{BCP} / \mathrm{PC}$ are more prone to hepatitis B-related acute-on-chronic liver failure and have a fatal outcome (10). In HCC patients from Asia and Africa, basal core mutations were detected. In Asia and the Pacific Ocean, BCP A1762T and G1764A mutations were more common. Precore mutations are common in the Mediterranean region and Romania is located close to this region. Genotype $\mathrm{C}$ is prevalent in Asia and the Pacific. Genotype D shows frequently precore mutations and is prevalent in the Mediterranean region and Southern Europe (11). In patients with HCC in Romania, we detected both core and precore mutations in tissue samples. Genotype D presents both BCP and PC mutations (28.3\%) more frequently compared to the mutation presenting PC only (21.8\%), in Romanian patients according to our study. The genotype D was detected in 60.5\% of our HBV patients, and we observed that genotype D was associated with both BCP and PC mutations. Previous studies indicated that high circulating HBV DNA levels are predictive of liver disease progression. Moreover, studies have recently reported that elevated HBV DNA levels in patients with cirrhosis increase the probability of mortality from non-HCC-related liver complications than low HBV DNA values (12). High-circulating HBV DNA levels were predictive of HCC development $(4,13,14)$.

In the present study, $60.53 \%$ of chronic carriers had an HBV DNA level of $\leq 10^{5} \mathrm{IU} / \mathrm{mL}$. However, only three patients from 73 with HCC had HBV DNA level $\leq 10^{5} \mathrm{IU} /$ $\mathrm{mL}$. In chronic B hepatitis patients with negative HBeAg was described in the PC region the mutation G1896A (4). These patients have continuous synthesize of HBV DNA at sufficient levels to cause constant liver damage with progression to cirrhosis (15-17). Patients with HBV load $\geq 10^{5}$ $\mathrm{IU} / \mathrm{mL}$ and infected with BCP and PC mutations have an increased risk of developing HCC, compared to those infected with WT. Detecting HBV viremia level is a criterion to assess infection risk progression to cirrhosis and HCC, and is used to detect patients requiring antiviral therapy to evaluate response to treatment and emergence of resistant mutants (11). Our study found differences between tissue and serum samples sequencing for HCC patients. Both BCP and PC mutations were detected in the tissue samples (75.3\%), and only PC mutation in the serum samples of our HCC patients (66.7\%), suggesting that BCP/PC mutant or hepatocytes harboring this mutant may be under immune selection and that such mutations may facilitate integration and tumor development. The presence of precore escape mutants should be considered in individuals who exhibit HBeAg negativity, HBsAg positivity, anti-HBe positivity, HBV DNA positivity, and elevated serum aminotransferase levels. However, precore variant is not uniformly pathogenic; thus, co-mutations or host factors presumably explain more virulent forms of precore mutant-associated disease $(18,19)$. Another common HBeAg variant is the core promoter mutant, characterized by point mutations in the promoter of both HBeAg mRNA and core protein mRNA. Core promoter mutants express less HBeAg through transcriptional downregulation. The most frequent core promoter mutation is the double A1762T and G1764A nucleotide exchange, which results in a substantial decrease in HBeAg expression but enhanced viral genome replication. Reduction of HBeAg expression is apparently mediated by reduced precore RNA transcription; whereas, the mechanism of enhanced replication is unclear. These virologic properties lead to enhanced pathogenicity of core promoter mutants in vivo. Enhanced replication capacity and reduced virion secretion may increase viral load in the liver, which triggers liver damage directly or indirectly through the immune response. Massive liver damage during acute infection leads to fulminant hepatitis. Damage during chronic infection increases hepatocyte turnover, induces fibrosis, and increases the chance of hepatocellular transformation and malignancy (20-22). Our study limitations were lack of long-term follow-up of subjects and liver biopsies were not performed in chronic carriers, a single baseline measurement for HBV DNA in chronic carriers and HCC patients. Fluctuations in serum HBV DNA occur especially during the exacerbation phases of the disease. Although some mutations statistically associated with HCC were found in this study, further studies are necessary to see whether these mutations predict the development of HCC in those who carry the mutation but do not yet have the associated disease. Future studies should also consider other viral mutations and the genetic makeup of the host. Association of core promoter mutations and liver cancer is also controversial; carefully conducted epidemiologic studies are needed to demonstrate a link between core promoter mutations and hepatocellular carcinogenesis $(23,24)$.

In summary, genotype $\mathrm{D}$ was the main genotype detect- 
ed in Romanian patients with chronic $\mathrm{HBV}$ and is associated with BCP A1762T/G1764A mutation, followed by the PC G1896A mutation and high HBV DNA load, suggesting an association between BCP mutations, high viral DNA load and hepatocarcinogenesis, consistent with previous reports. In our HCC-tissue HBV sequencing group we detected an increased number of both BCP and PC mutations, correlated with a high value of HBV DNA.

\section{Acknowledgements}

The authors are thankful to the Biosupply Company for their technical support, which made possible this study.

\section{Authors' Contributions}

Study concept and design: Ileana Constantinescu. Interpretation of data: Ileana Constantinescu, Andrei-Antoniu Dinu and Marius Niculescu. Drafting of the manuscript: Andrei-Antoniu Dinu. Critical revision of the manuscript for important intellectual content: Ileana Constantinescu and Andrei-Antoniu Dinu. Statistical analysis: Voicu Boscaiu.

\section{Funding/Support}

This study was supported in part by University of Medicine and Pharmacy "Carol Davila", Bucharest and the Fundeni Clinical Institute, Bucharest.

\section{References}

1. Tacke F, Gehrke C, Luedde T, Heim A, Manns MP, Trautwein C. Basal core promoter and precore mutations in the hepatitis B virus genome enhance replication efficacy of Lamivudine-resistant mutants. J Virol. 2004;78(16):8524-35.

2. Voiculescu M, Iliescu L, Ionescu C, Micu L, Ismail G, Zilisteanu D, et al. A cross-sectional epidemiological study of HBV, HCV, HDV and HEV prevalence in the SubCarpathian and South-Eastern regions of Romania. J Gastrointestin Liver Dis. 2010;19(1):43-8.

3. Tong MJ, Blatt LM, Kao JH, Cheng JT, Corey WG. Basal core promoter T1762/A1764 and precore A1896 gene mutations in hepatitis B surface antigen-positive hepatocellular carcinoma: a comparison with chronic carriers. Liver Int. 2007;27(10):1356-63.

4. Fang ZL, Ling R, Wang SS, Nong J, Huang CS, Harrison TJ. HBV core promoter mutations prevail in patients with hepatocellular carcinoma from Guangxi, China. J Med Virol. 1998;56(1):18-24.

5. Shi M, Zhang Y, Zhang J, Liu W, Xing L. Hepatitis B virus genotypes, precore mutations, and basal core promoter mutations in HBV-infected Chinese patients with persistently normal alanine aminotransferase and low serum HBV-DNA levels. Braz J Infect Dis 2012;16(1):52-6.

6. Yang HC, Chen CL, Shen YC, Peng CY, Liu CJ, Tseng TC, et al. Distinct evolution and predictive value of hepatitis $\mathrm{B}$ virus precore and basal core promoter mutations in interferon-induced hepatitis B e antigen seroconversion. Hepatology. 2013;57(3):934-43.

7. Yeh SH, Tsai CY, Kao JH, Liu CJ, Kuo TJ, Lin MW, et al. Quantifica- tion and genotyping of hepatitis $B$ virus in a single reaction by real-time PCR and melting curve analysis. J Hepatol. 2004 41(4):659-66.

8. Erhardt A, Blondin D, Hauck K, Sagir A, Kohnle T, Heintges T, et al. Response to interferon alfa is hepatitis B virus genotype dependent: genotype $A$ is more sensitive to interferon than genotype D. Gut. 2005;54(7):1009-13.

9. Enomoto M, Tamori A, Nishiguchi S. Hepatitis B virus genotypes and response to antiviral therapy. Clin Lab. 2006;52(1-2):43-7.

10. Ren X, Xu Z, Liu Y, Li X, Bai S, Ding N, et al. Hepatitis B virus genotype and basal core promoter/precore mutations are associated with hepatitis B-related acute-on-chronic liver failure without pre-existing liver cirrhosis. J Viral Hepat. 2010;17(12):887-95.

11. Ghabeshi S, Sharifi Z, Hosseini SM, Mahmoodian Shooshtari M Correlation between viral load of HBV in chronic hepatitis B patients and precore and Basal core promoter mutations. Hepat Mon. 2013;13(2).

12. Tong MJ, Blatt LM, Kao JH, Cheng JT, Corey WG. Precore/basal core promoter mutants and hepatitis B viral DNA levels as predictors for liver deaths and hepatocellular carcinoma. World J Gastroenterol. 2006;12(41):6620-6.

13. Ohata K, Hamasaki K, Toriyama K, Ishikawa H, Nakao K, Eguchi $\mathrm{K}$. High viral load is a risk factor for hepatocellular carcinoma in patients with chronic hepatitis B virus infection. J Gastroenterol Hepatol. 2004;19(6):670-5.

14. Chen CJ, Yang HI, Su J, Jen CL, You SL, Lu SN, et al. Risk of hepatocellular carcinoma across a biological gradient of serum hepatitis B virus DNA level. JAMA. 2006;295(1):65-73.

15. Chen BF, Liu CJ, Jow GM, Chen PJ, Kao JH, Chen DS. High prevalence and mapping of pre-S deletion in hepatitis B virus carriers with progressive liver diseases. Gastroenterology. 2006;130(4):1153-68.

16. Hunt CM, McGill JM, Allen MI, Condreay LD. Clinical relevance of hepatitis B viral mutations. Hepatology. 2000;31(5):1037-44.

17. Kao JH, Chen PJ, Lai MY, Chen DS. Basal core promoter mutations of hepatitis B virus increase the risk of hepatocellular carcinoma in hepatitis B carriers. Gastroenterology. 2003;124(2):327-34.

18. Yotsuyanagi H, Hino K, Tomita E, Toyoda J, Yasuda K, Iino S. Precore and core promoter mutations, hepatitis B virus DNA levels and progressive liver injury in chronic hepatitis B. J Hepatol. 2002;37(3):355-63.

19. Muroyama R, Kato N, Yoshida H, Otsuka M, Moriyama M, Wang Y et al. Nucleotide change of codon 38 in the X gene of hepatitis $B$ virus genotype $\mathrm{C}$ is associated with an increased risk of hepatocellular carcinoma. J Hepatol. 2006;45(6):805-12.

20. Liu CJ, Chen BF, Chen PJ, Lai MY, Huang WL, Kao JH, et al. Role of hepatitis B virus precore/core promoter mutations and serum viral load on noncirrhotic hepatocellular carcinoma: a case-control study. J Infect Dis. 2006;194(5):594-9.

21. Liu CJ, Chen BF, Chen PJ, Lai MY, Huang WL, Kao JH, et al. Role of hepatitis B viral load and basal core promoter mutation in hepatocellular carcinoma in hepatitis B carriers. J Infect Dis 2006;193(9):1258-65.

22. Zhang Q, Cao G. Genotypes, mutations, and viral load of hepatitis B virus and the risk of hepatocellular carcinoma: HBV properties and hepatocarcinogenesis. Hepat Mon. 2011;11(2):86-91.

23. Craxi A, Yurdaydin C. From viral pathobiology to the treatment of hepatitis B virus infection EASL Monothematic Conference (Istanbul, Turkey, October 6-8, 2005). J Hepatol. 2006;44(6):1186-95.

24. Papatheodoridis GV, Manesis EK, Manolakopoulos S, Elefsiniotis IS, Goulis J, Giannousis J, et al. Is there a meaningful serum hepatitis B virus DNA cutoff level for therapeutic decisions in hepatitis B e antigen-negative chronic hepatitis B virus infection? Hepatology. 2008;48(5):1451-9. 